CORRIGENDUM

doi:10.1038/nature10087

\title{
Diphthamide biosynthesis requires an organic radical generated by an iron-sulphur enzyme
}

Yang Zhang, Xuling Zhu, Andrew T. Torelli, Michael Lee, Boris Dzikovski, Rachel M. Koralewski, Eileen Wang, Jack Freed, Carsten Krebs, Steven E. Ealick \& Hening Lin

Nature 465, 891-896 (2010)

In this Article, the following studies, which led to the identification of diphthamide structure and its biosynthetic genes, should have been cited $^{1-5}$. Reference 1 reports the presence of an unusual amino acid at the ADP-ribosylation site of elongation factor 2 (EF2); ref. 2 reports the properties of the modified residue in EF2 and proposed the name diphthamide; ref. 3 reports the structure of diphthamide; ref. 4 describes work to suggest that the 3-amino-3-carboxypropyl group of diphthamide come from $S$-adenosyl methionine; and ref. 5 reports the identification of yeast mutants that are defective in diphthamide biosynthesis and proposes the biosynthetic pathway.

1. Robinson, E. A., Henriksen, O. \& Maxwell, E. S. Elongation factor 2. Amino acid sequence at the site of adenosine diphosphate ribosylation. J. Biol. Chem. 249, 5088-5093 (1974).

2. Van Ness, B. G., Howard, J. B. \& Bodley, J. W. ADP-ribosylation of elongation factor by diphtheria toxin: isolation and properties of the novel ribosyl-amino acid and its hydrolysis products. J. Biol. Chem. 255, 10717-10720 (1980).

3. Van Ness, B. G., Howard, J. B. \& Bodley, J. W. ADP-ribosylation of elongation factor 2 by diphtheria toxin: NMR spectra and proposed structures of ribosyl-diphthamide and its hydrolysis products. J. Biol. Chem. 255, 10710-10716 (1980)

4. Dunlop, P. C. \& Bodley, J. W. Biosynthetic labelling of diphthamide in Saccharomyces cerevisiae. J. Biol. Chem. 258, 4754-4758 (1983).

5. Chen, J. Y., Bodley, J. W. \& Livingston, D. M. Diphtheria toxin-resistant mutants of Saccharomyces cerevisiae. Mol. Cell. Biol. 5, 3357-3360 (1985). 